\title{
ELECTROHYDRAULIC VIBRATION EXCITED BY 2D VALVE
}

\author{
Jian RUAN *, R. BURTON**, Sheng LI ${ }^{*}$ and Faming ZHU* \\ *The MOE Key Laboratory of Mechanical Manufacture and Automation \\ Zhejiang University of Technology \\ Hangzhou, 310014, China \\ (E-mail:wxmin@mail.hz.zj.cn) \\ **Department of Mechanical Engineering \\ University of Saskatchewan, Saskatoon, Canada
}

\begin{abstract}
The frequency bandwidth of the electrohydraulic vibrator conventionally constructed by a servo valve and a hydraulic cylinder or a motor is to a large extent limited to fairly narrow range by the dynamic response capability of the servo valve. A 2D electrohydraulic valve is thus developed to enhance the working frequency range to a new high level. In this $2 \mathrm{D}$ valve, the continuous rotary motion of the spool is used to create alternated variation of the valve port areas, while the linear motion of the spool is to control the peak value of the valve port areas. The frequency excited by $2 \mathrm{D}$ valve is proportional to the rotary speed of the spool and thus the high-frequency can easily be achieved by increasing the rotary speed of the spool well lubricated in hydraulic oil. Experiments have been carried out to the vibrator loaded by an elastic force coming from the deformation of a frame work and the wave forms of the cylinder piston was measured. It is demonstrated that the ascent and descent slopes of the wave form show some inconsistency which alleviates with the reduction of valve linear opening. The phenomenon is explained to be caused by the changing direction of the elastic force. Nevertheless, the wave from is close to a sinusoidal one. It is concluded the development of the $2 \mathrm{D}$ valve does point out an access to the high-frequency excitation of the hydraulic vibration.
\end{abstract}

\section{KEY WORDS}

Two-dimensional control valve; Electrohydraulic servo system,Hydraulic vibrator

\section{INTRODUCTION}

Vibration is a phenomenon commonly met in mechanical engineering practice. When the frequency of the external excited vibration comes close to the natural frequency of a mechanical structure, resonance occurs, which can be very destructive to the whole mechanical system.
Vibrating test therefore remains an important and objective means to determine the performance of a mechanical system, or part of it, working under vibrating condition $^{[1]}$. In the vibration tests, the vibrating environment is usually created artificially with a vibrator, which is driven by either mechanical power or electrical power or hydraulic power ${ }^{[2]}$. In the case of heavy-power 
demanded, hydraulic vibrator is commonly used. The conventional hydraulic vibrator is actually a hydraulic actuator, either a cylinder or a motor, controlled by a servo valve. By feeding to the coil of servo valve with a wave form of current in respect to the desired vibrating wave form, the cylinder piston or motor rotor will makes reciprocating motion driven by alternative flow rate into or out of its working chambers created by the servo valve. The reciprocating piston further results in a vibration of loads connected on cylinder rod or motor axis. The bandwidth of the hydraulic vibrator so constructed is greatly restrained by the dynamic performance of the servo valve ${ }^{[3]}$, which is difficult to be enhance to a very high level.

Therefore, searching for a new excitation method for hydraulic vibrator always remains particularly significant in the design of the hydraulic vibrator with wide bandwidth. A promising solution is to apply a new control valve called a two-dimensional or " $2 \mathrm{D}$ " valve ${ }^{[4]}$. In the $2 \mathrm{D}$ valve, both rotary and linear motions of a single spool are exploited to control the frequency and the amplitude independently. Since the frequency of the vibrator is proportional to the continuous rotary speed of the spool, it can be enhanced to a large extent by increasing the rotary speed of the spool, which is well lubricated in hydraulic oil.

\section{HYDRAULIC VIBRATOR EXCITED BY 2D VALVE}

The operating principle of the proposed hydraulic vibrator excited $2 \mathrm{D}$ valve is illustrated by Figure 1 . It is consists of a symmetrical cylinder and a $2 \mathrm{D}$ valve. The spool of the $2 \mathrm{D}$ valve is continuously driven by an electric motor. The pressurised oil into and out of the cylinder chambers is achieved using a series of grooves asymmetrically distributed on the spool lands, which create an alternatively varied valve port area between the grooves on the spool lands and the rectangular windows on the sleeve as the spool rotates. This can be clearly seen through the cross-sections of spool lands shown in Figure $1 \mathrm{~b}$.

If the central angle between two adjoining grooves is $\beta$, then geometrically, the groove of meter-in valve port has an $\beta / 2$ angle difference with respect to the meter-out valve port. Such an arrangement will cause a $180^{\circ}$ phase angle difference between the wave forms of the meter-in valve port and the meter-out valve port. As a result each of the bi-directional cylinders is a hydraulic bridge with a $180^{\circ}$ phase angle difference between the chambers. As the spool of the $2 \mathrm{D}$ valve makes a rotary motion the pressure inside the two chambers of the cylinder will change alternatively and the piston is subsequently driven in a reciprocating motion (see Figures 1(a) and (b)). The amplitude of the reciprocating motion or the amplification is controlled by the linear motion of the spool, through which the area of the valve ports between the grooves on the spool land and the windows on the sleeve can be adjusted. Obviously, the frequency of the reciprocating motion of the cylinder controlled by the $2 \mathrm{D}$ valve is proportional to the rotary speed of the spool $\omega$ and the number of the grooves on a single spool land $Z$

$$
f=Z \omega /(2 \pi)
$$

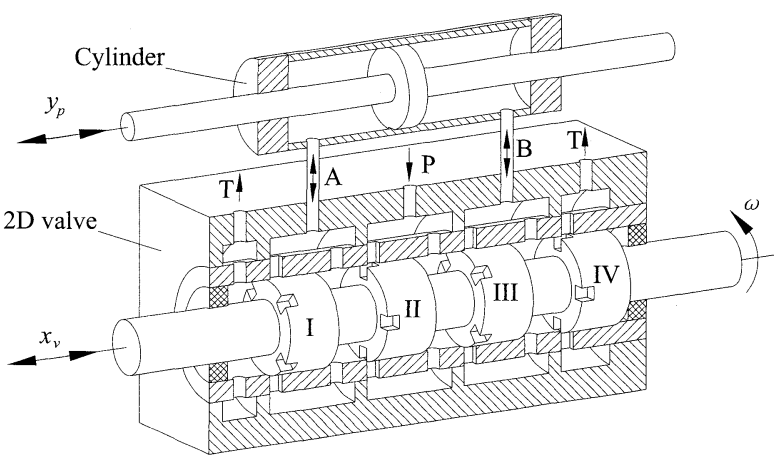

(a)

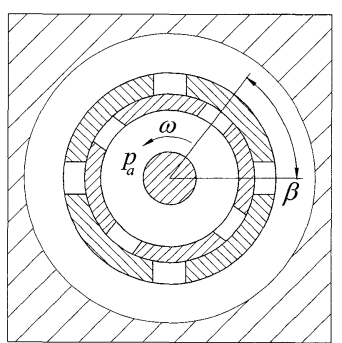

I

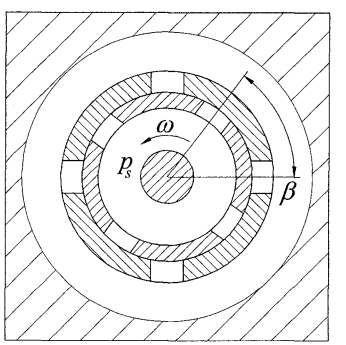

III

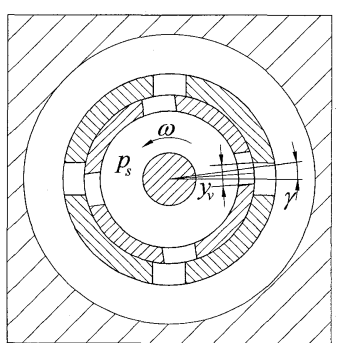

II

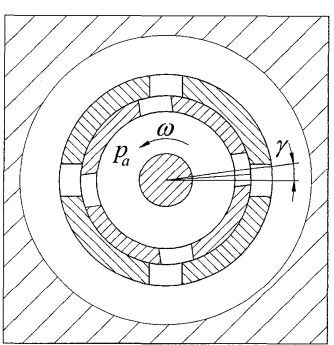

IV (b)

Figure 1 Hydraulic vibrator controlled by a $2 \mathrm{D}$ valve

The 2D valve used in the electrohydraulic vibration exciter has a spool with four lands labelled in I, II, III and IV in sequence from left to right in the Figure 1a. These four spool lands are divided into two groups. The spool lands I and II form one group and the spool lands III and IV form the other. On each spool land a number of grooves were asymmetrically distributed in 
peripheral direction to coordinate with the windows on the sleeve such that valve ports could be created. The valve ports formed on the spool lands I and II, served as meter-out and meter-in orifices respectively and as a result act as a hydraulic resistance bridge to vary the pressure inside one of the chamber of the double acting cylinder. The valve ports formed on the spool lands III and land IV form another hydraulic resistance bridge to control the pressure inside the other chamber of the cylinder. The numbers of the grooves on these four spool land are the same and the central angle of the adjacent grooves $\beta$ is equal to four times that of the grooves $\theta_{0}$, i.e., $\beta=4 \theta_{0}$. The phase angle of the grooves on each spool land is specially arranged. The phase angle of the grooves on the spool lands I and III are the same and the phase angle of the grooves on the spool lands II and IV are the same; however, the phase angle of the grooves on spool land I (or land III) has a value of $2 \theta$ (or $\beta / 2$ ) with respect to the grooves on spool land II (or land IV). The area of the valve port $A_{v I}-A_{v I I}$ vs. rotary angle $\gamma$ is given in Figure 2. It approximates a triangular wave form and its peak value varies with linear valve port opening $x_{v}$.

\section{EXPERIMENTAL SYSTEM AND RESULTS}

The hydraulic vibrator controlled with 2D valve designed for experiment is shown in Figure 3.

The rotary motion of the spool is driven with a variable $\mathrm{AC}$ servo motor while the linear motion of the spool is actuated and positioned the same way of the direct actuated digital valve ${ }^{[6]}$. In this way, the frequency and the amplitude of the vibration wave output from the cylinder are varied independently via the two independent input signals to the $2 \mathrm{D}$ valve controller. The frequency range of the vibrator is to a large degree constrained by the rotary speed of the spool of the 2D valve. The rotation of the $\mathrm{AC}$ servo motion is transmitted to the axis of the spool through a gear chain with an effective speed amplification of four. The actual frequency range for the experimental system was $0 \sim 800 \mathrm{~Hz}$.

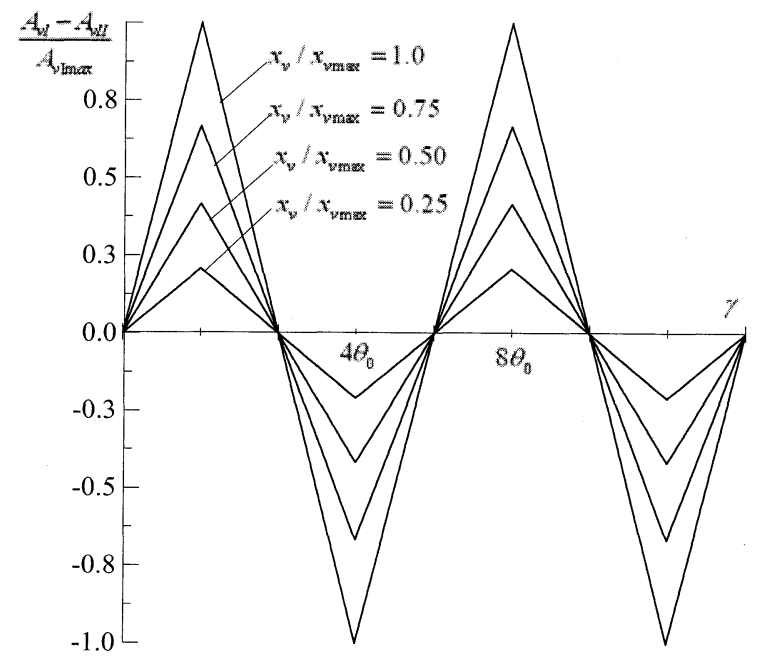

Figure 2 Area of the valve port $A_{v I}-A_{v I I}$

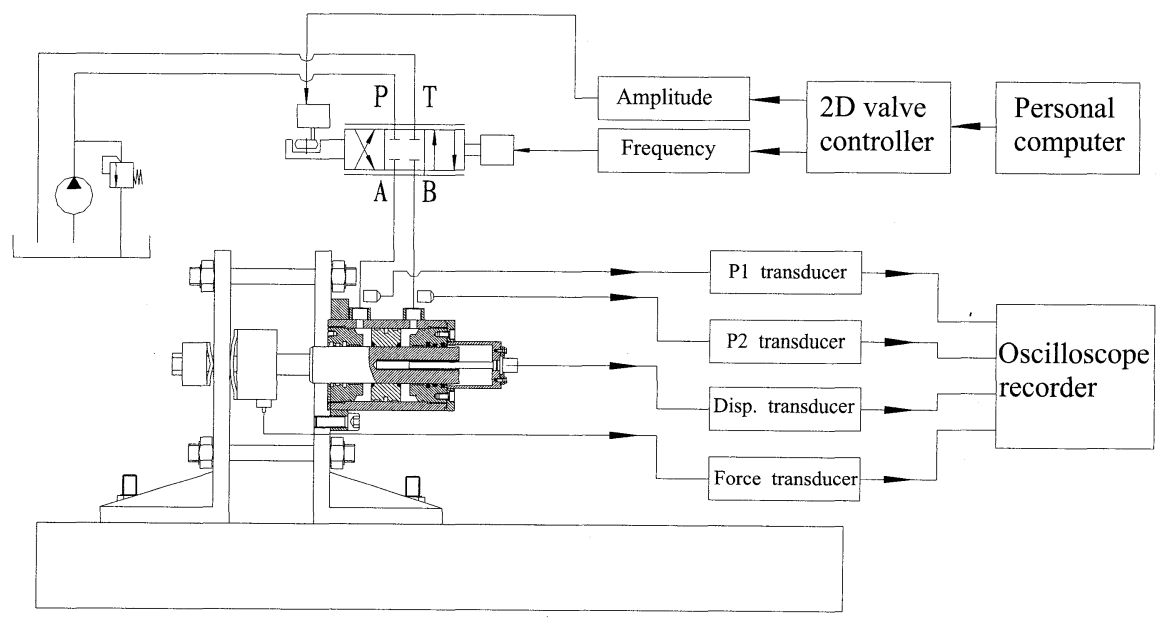

Figure 3 Schematic of the experimental system

Two pressure transducers were used to measure the pressure in each chamber of the cylinder. A force cell was placed between the end of cylinder rod and the rigidly connected frame. The system was therefore a rigid system with a very high spring constant. A displacement transducer was mounted inside of the 
cylinder rod to measure the displacement of the piston with respect to the frame in an axial direction of the cylinder. A calibration of the deformation vs. hydrostatic force output by cylinder was carried out using a laser displacement meter (manufactured by Keyance, Japan) with an error less than 0.6 micrometer; the calibration curve is given in Figure 4. It can be observed from the Figure 4 that the relationship between the deformation and the exerted hydrostatic force is fairly linear, which establishes that the deformation of the frame was in elastic range-- This ensured that the deformation wave forms of the frame vs. the hydraulic force and piston displacement of the piston were identical. Therefore, the output vibration is presented hereafter as the output force from the force cell.

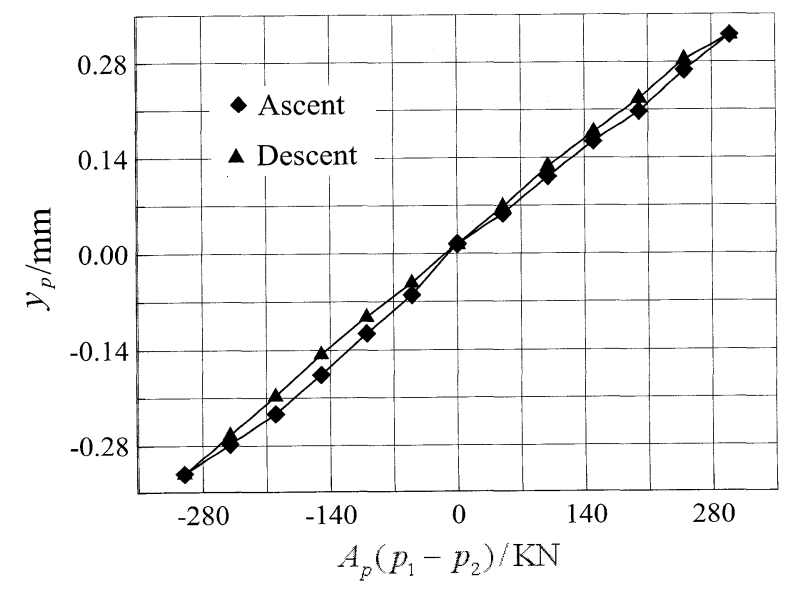

Figure 4 Calibration of deformation of frame work vs. hydrostatic force.

The measured wave forms in the low frequency domain range $(10$ to $200 \mathrm{~Hz})$ are given in Figure 5. It is observed that the measured waves demonstrate the different slopes during ascent and descent which was consistent with the simulation results. The difference was associated with the changing direction of the elastic force of the frame work deformation with respect to the hydrostatic force.

As the spool is rotated, the area of the valve port opening increases and decreases alternatively at the same rate. The process can be approximated as a triangular wave form with the ascent slope being the same as the descent, as shown in Figure 2. This triangular shape is not reflected in the output displacement or force wave form because of the changed direction of the elastic force. With reference to Figures 5 (a), (b), a discussion to the motion of the cylinder piston with the variation of the valve port opening will take an insight into the phenomenon. When the piston is at negative maximum and the speed is zero, the rotary valve ports are all closed at the point, see Figure 2 and the flow rate is at zero). As the spool rotates, the valve port opening area increases to maximum, pressurized oil is directed to the left cylinder chamber and drained from the right cylinder chamber to tank. This produces a hydrostatic force driving the piston to move in the right hand direction. At this point, the spring force acts as a restoring force, in the same direction as the hydrostatic force. The spring load essentially becomes an "overrunning load. Thus, the curve has a steeper slope. When the valve opening and flow rate reach their maximum and the speed of the piston is at its peak value. As the rotation of the spool continues, the valve port opening area varies from the maximum value to zero, the direction of the hydrostatic remains the same as the proceeding process, but the elastic load turns into a compressed spring, which consumes part of the hydrostatic force. Thus, the curve becomes a smaller slope. When the valve opening and flow rate both drop to zero, the displacement of piston reaches its peak value. A similar reasoning can also be applied to the following process. It should be noted that at the same valve port opening the value of the slope of the positive curve equals to the absolute value of the slope of the negative curve.

The ascent process and descent processes in the wave form of the output displacement $y_{p}$ or force $K y_{p}$ display some inconsistency because of the changing of the elastic load force, which becomes more significant at the critical valve linear opening $x_{v c}$. As the valve linear opening $x_{v}$ decreases, the amplitude of the wave form of the output displacement or force will decrease. Concurrently, the load pressure also decreases, but the pressure drop across the valve port will increase since the system pressure $p_{s}$ is fixed. As a result, the flow rate is less affected by the pressure fluctuation, but is more dependent on the cyclic opening area variation of the rotary valve port. In this case, the change of the flow rate tends to be in the form of a triangular wave and the ascent and descent rates in the wave form of the output displacement or force tend to be identical to each other or to the flow form.

\section{CONCLUSION}

A new approach for the excitation of the hydraulic vibration is presented using a $2 \mathrm{D}$ valve which extends the frequency range substantially. In $2 \mathrm{D}$ valve, the rotary motion of the spool coordinates the grooves of the spool land with the windows on the sleeve and alternates flow into and out of the chambers of the hydraulic cylinder, thus exciting the piston (rotor) to output a vibration. The spool's linear motion is used to adjust the peak flow rate and thus the amplitude of the output vibration. The frequency of the vibration is excited by the $2 \mathrm{D}$ valve and is related to the rotary speed of the spool. Thus the potential to extend the frequency of the hydraulic vibrator to even higher levels exists by 
increasing the rotary speed of the spool. The experimental results show that in the low frequency domain ascending and descending slopes of the output force waveforms show some differences which become more pronounced at spool displacements above the critical valve linear opening area. As the working frequency passes beyond the low frequency domain, the vibration excited by the $2 \mathrm{D}$ valve is mainly influenced by the hydraulic resonance.

It was concluded that the proposed $2 \mathrm{D}$ valve and actuator have successfully extended the frequency range of the electrohydraulic vibration exciter.

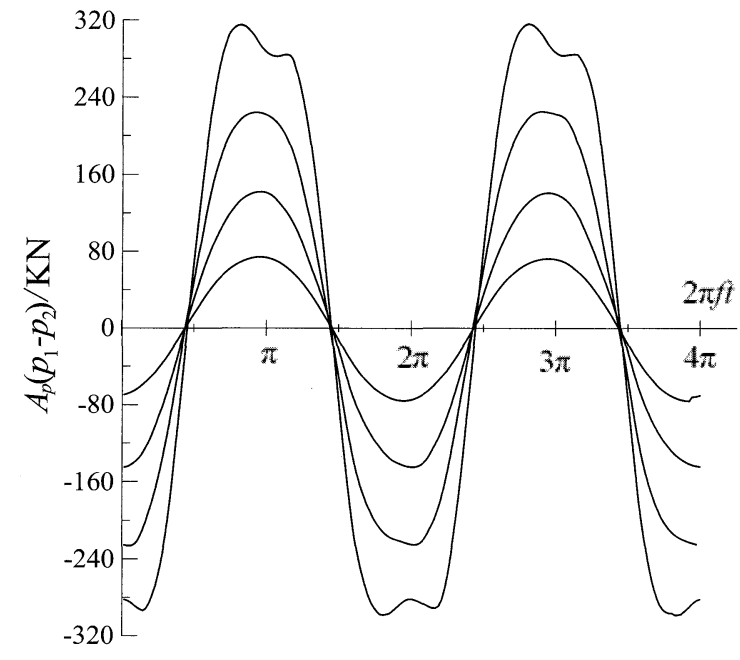

(a) $f=10 \mathrm{~Hz}$

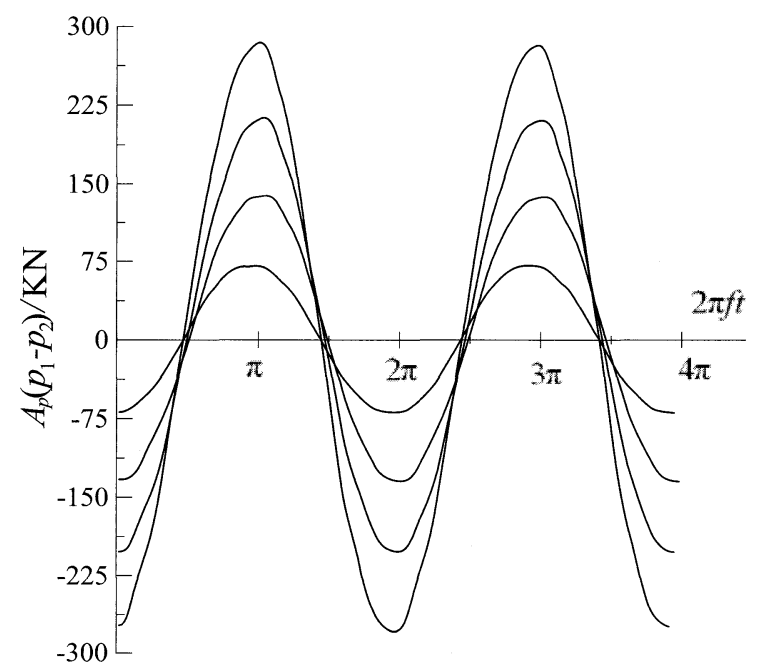

(c) $f=100 \mathrm{~Hz}$

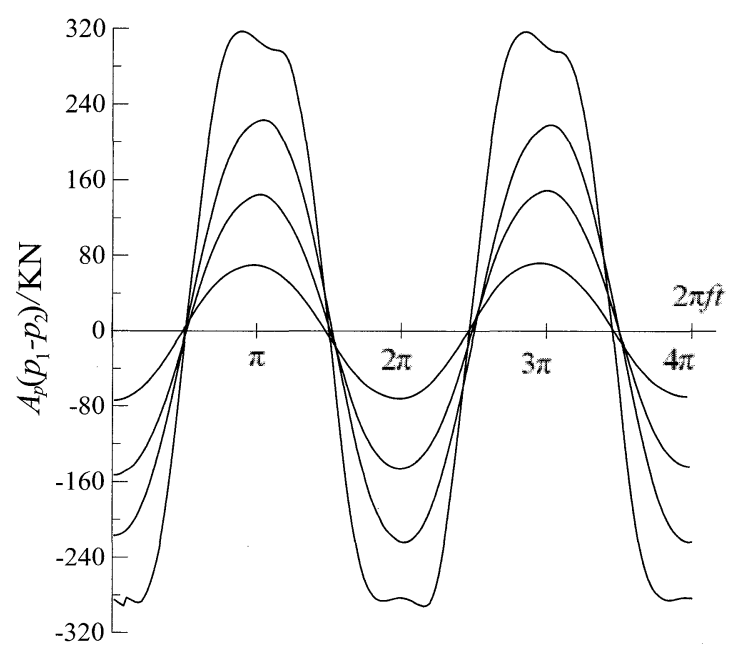

(b) $f=40 \mathrm{~Hz}$

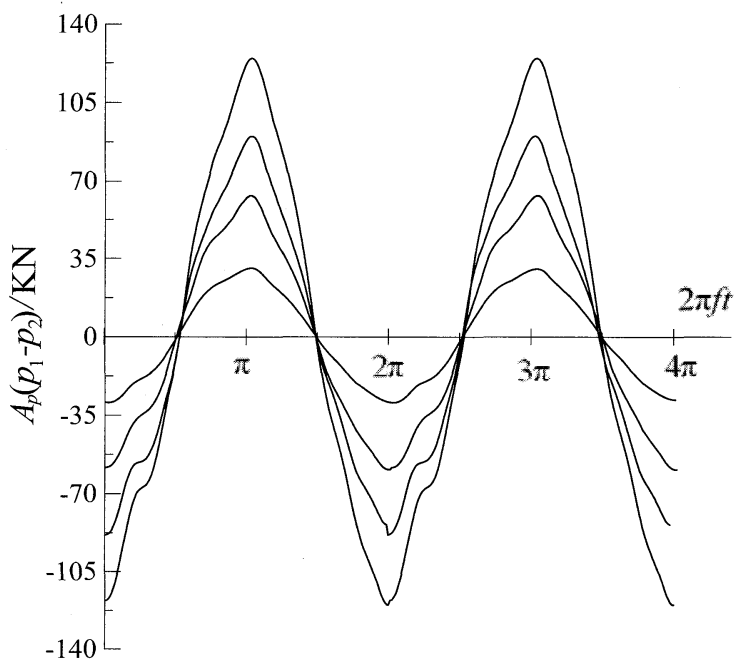

(d) $f=200 \mathrm{~Hz}$

Figure 5 Wave forms in the lower frequency domain

\section{ACKNOWLEDGEMENTS}

The authors would like to offer their gratitude to The National Foundation of Natural Sciences (No. 50675204), the Foundation of Natural Sciences of Zhejiang Province
(No.Y106165) and the Foundation of the MOE Key Laboratory of Mechanical Manufacture and Automation who sponsored the project. Gratitude is also extended to the Watch Manufacturing Factory of Hangzhou who helped machine the testing elements. 


\section{REFERENCES}

2. Lang, Geore Fox, Electrodynamic shaker fundamentals, S V Sound and Vibration, v 31, n 4, Apr, 1997,pp.14 21.

3. SU Donghai, CUI Xiao, WU Xihong, Analysis and Simulation of Dynamic Properties of High Frequency Hydraulic Vibration, 7th International Conference on Progress of Machining Technology, 2004,pp.918 922.

4. Jian Ruan and Richard Burton, Bulk Modulus of Air Content Oil in a Hydraulic Cylinder. ASME,
1. K. G.. McConnell, Vibration Testing---Theory and Practice, New York, 1995.

IMECE2006-15854, Fluid Power Control System Technology, Vol.7, 2006.

5. Merritt, H. E., Hydraulic Control Systems, John Wiley \& Sons, New York, 1967,pp.67 86.

6. J. Ruan R. Burton and Ukrainez, Direct Actuated Digital Servo Valve, The Ninth Scandinavian International Conference on Fluid Power, SICF'05, June 1-3, 2005, Linköping ,pp.450-461. 